\title{
Diseño y evaluación de un laboratorio virtual de vectores en 3D
}

Vanessa P. Cuenca-Gotor ${ }^{\mathrm{a}, \mathrm{b}}$, Marcos H. Giménez ${ }^{\mathrm{a}, \mathrm{c}}$, José A. Gómez-Tejedor ${ }^{\mathrm{a}, \mathrm{d}}$, Rosa Martínez-Sala $^{\mathrm{a}, \mathrm{e}}$, Juan A. Monsoriu, ${ }^{\mathrm{a}, \mathrm{f}}$, Isabel Salinas ${ }^{\mathrm{a}, \mathrm{g}}$ y Juan A. Sans ${ }^{\mathrm{a}, \mathrm{h}}$

${ }^{a}$ Departamento de Física Aplicada, Universitat Politècnica de València, Camino de Vera s/n, 46022 Valencia, Spain, ${ }^{\mathrm{b}}$ vacuego@ fis.upv.es, ${ }^{\mathrm{c}} \mathrm{m}$ mgimene@ fis.upv.es, ${ }^{\mathrm{d}}$ jogomez@ fis.upv.es,

ermsala@fis.upv.es, ${ }^{\mathrm{f}}$ jmonsori@ $@$ fis.upv.es, ${ }^{\mathrm{g}}$ isalinas@ fis.upv.es y ${ }^{\mathrm{h}}$ juasant2@ upvnet.upv.es

\begin{abstract}
In Physics, many quantities are vectors, and their use requires typical operations such as: addition, substraction, scalar multiplication, scalar product (dot product), vector product (cross product) and scalar triple product. This is a very basic topic in all General Physics courses for Engineering degrees. However, we have detected that some students lack of a deep understanding of vector operations and their properties.

In this work, we present a virtual laboratory (developed using the tool "Easy Java Simulations") for the study and understanding of these topics. The user can introduce the components of the input vectors and gets a $3 D$ representation, which can be scaled and rotated for a better visualization. Any of the aforementioned operations can be selected, and the result is shown both numerically and graphically. The user can also modify any represented vector. In this way, the virtual lab provides a real time visualization of how the change affects the result. The possibility of limiting the changes to either magnitude or direction is also included.

The efficiency of the virtual laboratory has been tested analyzing the results obtained in two groups of students (virtual laboratory vs. traditional resources). A satisfaction survey has been also carried out.
\end{abstract}

Keywords: virtual laboratory, Physics, Easy Java, vector analysis.

\section{Resumen}

En Física, un gran número de magnitudes son vectores, y su uso requiere operaciones típicas tales como: suma, resta, multiplicación por un escalar, producto escalar, producto vectorial y producto mixto. Se trata de conceptos básicos en todos los cursos de Física General para grados en Ingeniería. Sin embargo, algunos estudiantes carecen de una comprensión profunda de las operaciones vectoriales y sus propiedades.

En este trabajo presentamos un laboratorio virtual (desarrollado utilizando la herramienta "Easy Java Simulations") para el estudio y comprensión de estos conceptos. El usuario puede introducir las componentes de los vectores de entrada y obtiene una representación $3 D$, que puede escalar y girar para una mejor visualización. Se puede seleccionar cualquiera de las operaciones 
antes mencionadas, y se muestra el resultado tanto numérica como gráficamente. El usuario puede también modificar cualquiera de los vectores representados. De esta forma, el laboratorio virtual proporciona una visualización en tiempo real de cómo el cambio afecta al resultado. También se incluye la posibilidad de modificar únicamente el módulo o la orientación.

Para comprobar la eficiencia del laboratorio virtual, se han analizado los resultados obtenidos por dos grupos de estudiantes (laboratorio virtual vs. recursos tradicionales). También se ha realizado una encuesta de opinión.

Palabras clave: laboratorio virtual, Física, Easy Java, análisis vectorial.

\section{Introducción}

En los temas de Mecánica que se imparten en las asignaturas de Física de primer curso, los estudiantes han de adquirir conceptos básicos que pueden resultar de difícil comprensión, o complicados de dominar. Los laboratorios virtuales son una de las herramientas más versátiles de que pueden disponer los estudiantes para facilitar este aprendizaje (Vidaurre, 2002). En primer lugar, ponen el foco en el aprendizaje activo, utilizando unas herramientas con las que los alumnos de hoy día están ampliamente familiarizados, y con significativas ventajas en la educación, como la interactividad y la respuesta dinámica (Jimoyiannis, 2001).

En segundo lugar, los laboratorios virtuales no tienen por qué limitarse a realizar más rápido un ejercicio tipo, sino que pueden permitir el control de las variables de un proceso, y por tanto analizar su influencia, mostrando además las representaciones necesarias (imágenes, gráficas, animaciones) para asimilar los conceptos y relaciones subyacentes. De esta forma, constituyen una herramienta de apoyo para el desarrollo de competencias transversales como la comprensión e integración de conceptos, análisis y resolución de problemas y pensamiento crítico, principalmente. En este contexto, los procesos de aprendizaje basados en laboratorios virtuales han demostrado proporcionar varias ventajas (Esquembre, 2002), aunque su eficacia pedagógica está condicionada a una integración completa en el proyecto docente global (Steinberg, 2000).

En este trabajo presentamos tanto el diseño como la evaluación de un nuevo laboratorio virtual (http://personales.upv.es/mhgimene/lab/vectores.html) para el estudio y comprensión de las operaciones vectoriales y sus propiedades tales como: suma, resta, multiplicación por un escalar, producto escalar, producto vectorial y producto mixto. El laboratorio virtual desarrollado proporciona una visualización en tiempo real de dichas operaciones y muestra cómo el cambio de los parámetros implicados afecta al resultado.

El presente laboratorio virtual se ha desarrollado utilizando la herramienta "Easy Java Simulations" (EJS) (Esquembre, 2004; http://www.um.es/fem/Ejs), basada en Open Source

(cc) EY-NC-ND 2017, Universitat Politècnica de València

Congreso IN-RED (2017) 
Physics (Christian, 2001). EJS es una herramienta de gratuita que permite crear applets interactivos en Java o JavaScript (HTML5), principalmente para fines de enseñanza o aprendizaje. El laboratorio virtual desarrollado es independiente de la plataforma, por lo que puede visualizarse utilizando cualquier navegador web.

Para la evaluación del laboratorio desarrollado se han realizado encuestas a los estudiantes a través de la plataforma PoliformaT (https://poliformat.upv.es), con el objetivo de conocer su opinión, la valoración de la utilidad y claridad del laboratorio virtual, el grado de desarrollo de las competencias transversales, y dar lugar así a un proceso de retroalimentación. También se han comparado los resultados de aprendizaje en dos grupos piloto, uno en el que se ha implementado el presente laboratorio virtual y otro en el que ha utilizado una metodología tradicional.

Con el objetivo de valorar la influencia del uso del laboratorio virtual de Vectores sobre el aprendizaje de los alumnos, durante el presente curso académico (16/17) se han seleccionado dos grupos de control, pertenecientes al primer curso de dos títulos de grado de la Escuela Técnica Superior de Ingeniería del Diseño de la Universitat Politècnica de València, y a dos asignaturas cuyos programas incluyen el tema de Vectores. En el grupo de la asignatura "Complementos de Física" del grado en Ingeniería Mecánica se impartió esta tema de forma clásica, y en el grupo de la asignatura "Física Básica" del grado en Ingeniería Eléctrica se utilizó el laboratorio virtual.

\section{Objetivos}

Los principales objetivos del laboratorio virtual son:

- Visualizar un vector, comprender qué son sus componentes en un sistema ortonormal de referencia, y comprobar que esas componentes determinan el vector.

- Asimilar las operaciones básicas entre vectores, y entre vector y escalar, tanto analítica como gráficamente, y comprender sus propiedades.

- Que el estudiante compruebe que sabe realizar estas operaciones.

- Visualizar cómo la modificación de un operando afecta al resultado de las diferentes operaciones.

- Comprender las relaciones entre diversas propiedades geométricas y las operaciones entre vectores.

\section{Desarrollo de la innovación}

El laboratorio virtual de vectores (con opción de elegir el idioma entre español, valenciano e inglés) permite que el estudiante introduzca las componentes de tres vectores y un escalar, y que seleccione una de las seis operaciones disponibles (suma, resta, producto escalar, producto vectorial y producto mixto de vectores, además de multiplicación de escalar y vector). Los vectores y el resultado de la operación (cuando éste sea un vector) se muestran 
en una representación 3D en la que el usuario puede modificar de forma interactiva tanto el punto de vista como la escala.

Como alternativa a la introducción manual de componentes de un vector, el usuario puede modificarlas arrastrando su extremo en el visor. De este modo, puede observar de forma interactiva cómo afectan las modificaciones al resultado de la operación seleccionada. Se puede elegir que el arrastre del extremo del vector sea libre, que afecte únicamente a su módulo, o que cambie solamente su orientación. Además, se dispone de botones de copiar y pegar para, por ejemplo, utilizar el resultado de una operación como operando de otra.

El laboratorio incluye botones para visualizar diversas propiedades geométricas de interés: el ángulo que forman dos vectores, la proyección de uno sobre otro, el paralelogramo que definen, o el paralelepípedo constituido por tres vectores.

La Figura 1 muestra una captura del laboratorio virtual. El visor presenta una representación 3D de un sistema ortonormal de referencia, junto con los vectores y elementos adicionales. La representación es totalmente interactiva, pudiendo arrastrar con el ratón el extremo de un vector para modificarlo, o cualquier otro punto para cambiar el punto de vista de la perspectiva. La escala se controla con la deslizadera "Zoom" ubicada en el panel "Visualización".

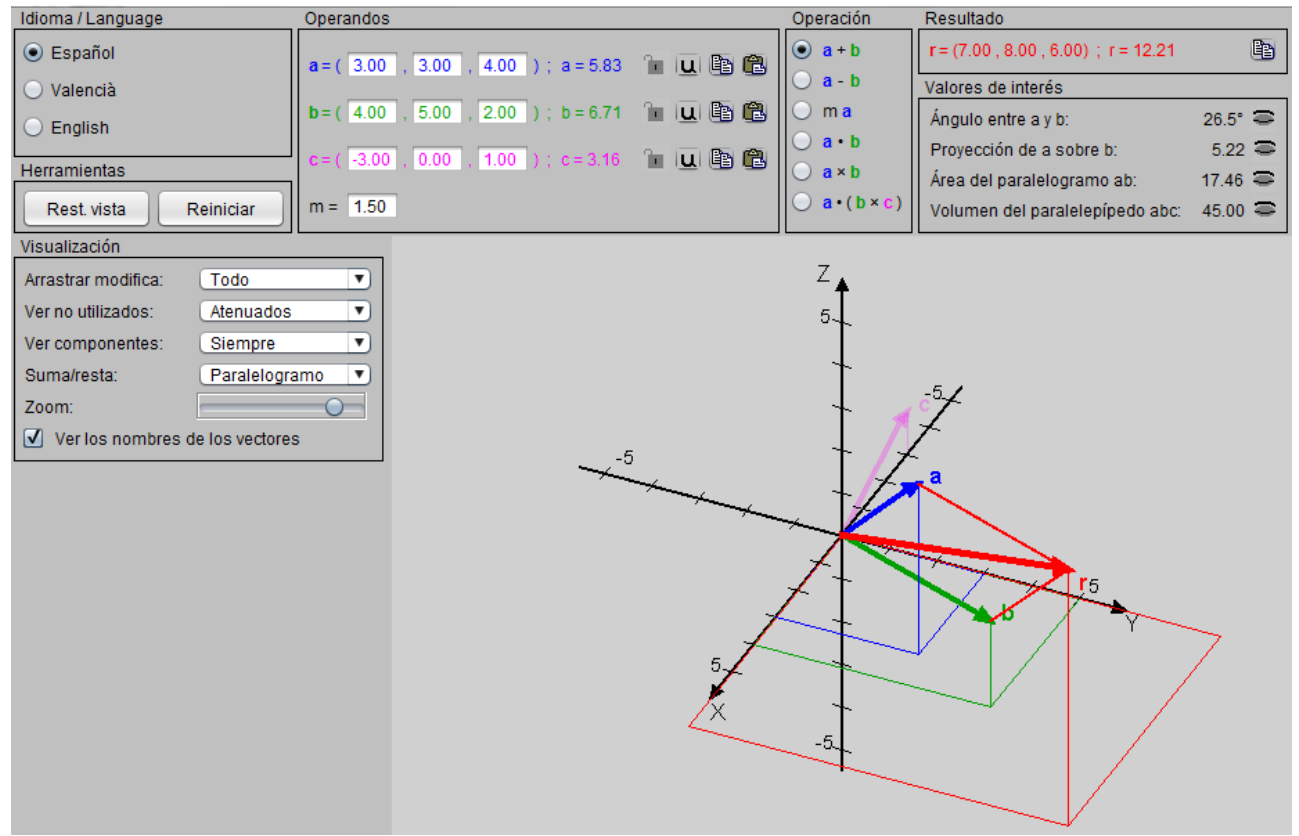

Fig. 1. Pantalla inicial de la aplicación.

Dado que el puntero del ratón se desplaza en dos dimensiones, no es posible modificar por arrastre las tres componentes de un vector de forma simultánea. Por esta razón, sólo varían dos de ellas (cuáles en concreto depende del punto de vista de la perspectiva). Sin embargo, es posible controlar una única componente cualquiera pulsando la correspondiente tecla. Por ejemplo, si se arrastra el extremo de un vector mientras se mantiene pulsada la tecla X, sólo varía esta componente.

(cc) EY-NC-ND 2017, Universitat Politècnica de València 
El panel "Operandos" (Figura 2) permite al estudiante introducir valores numéricos concretos para las tres componentes de cualquier vector, y muestra automáticamente el correspondiente módulo a la derecha. Al escribir en un campo numérico, su color de fondo es amarillo. La tecla INTRO permite validar la entrada, pasando el fondo a ser blanco. Si en lugar de eso pasa a ser rojo, significa que la entrada no es un número válido. También se puede introducir el valor de un escalar para utilizarlo en la operación de multiplicación por un vector. Cabe destacar además que cada vector tiene asociado un color (azul, verde, violeta) que permite identificarlo rápidamente, tanto en el visor como en los diferentes paneles.

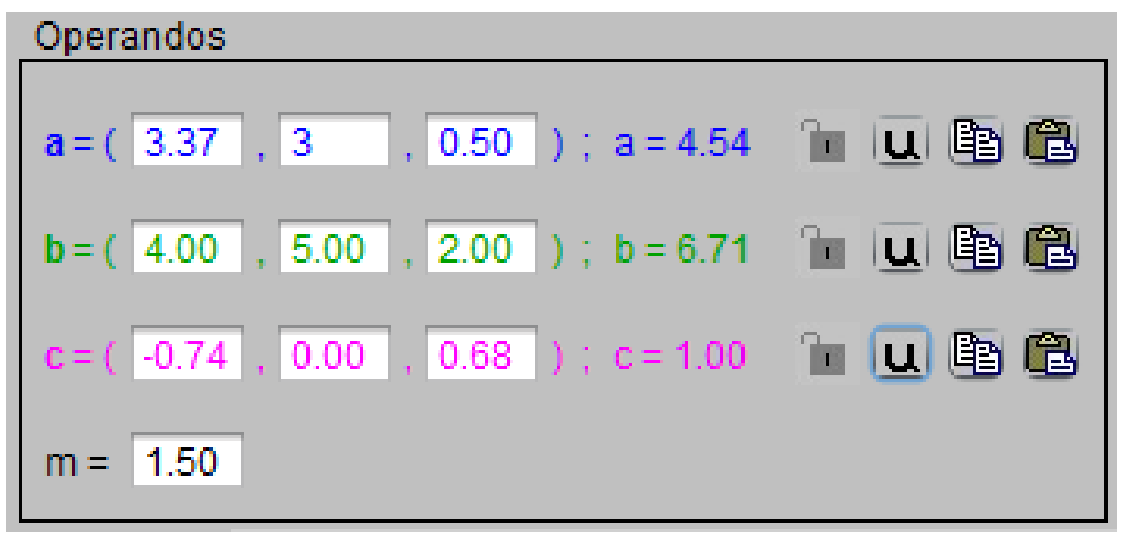

Fig. 2. Detalle del panel "Operandos".

El laboratorio virtual proporciona diferentes botones de control. Además de los de copiar y pegar ya mencionados, están los de bloquear (no se puede modificar las componentes, ni en el visor ni el panel) y los de transformar en vector unitario.

El panel "Operación" (Figura 3) permite elegir una de las seis disponibles. Cuando el resultado es un escalar, su valor se muestra en el panel "Resultado". En caso de que el resultado sea un vector, el panel muestra sus componentes y módulo, y también aparece representado en rojo en el visor. Como ejemplo, la Figura 4 muestra el resultado del producto vectorial de dos vectores.

\begin{tabular}{|c|c|c|}
\hline Operación & \multicolumn{2}{|l|}{ Resultado } \\
\hline \multirow{6}{*}{$\begin{array}{l}\text { a+b } \\
a-b \\
m a \\
a \cdot b \\
a \times b \\
a \cdot(b \times c)\end{array}$} & Valor $=29.48$ & 舄宜 \\
\hline & Valores de interés & \\
\hline & Ángulo entre a y b: & $14.5^{\circ} \boldsymbol{\varnothing}$ \\
\hline & Proyección de a sobre b: & $4.40 \overline{\boldsymbol{\sigma}}$ \\
\hline & Área del paralelogramo ab: & $7.64 \equiv$ \\
\hline & Volumen del paralelepípedo abc: & $0.69 \sqsupseteq$ \\
\hline
\end{tabular}

Fig. 3. Detalle de los paneles "Operación", "Resultado" y "Valores de interés". 


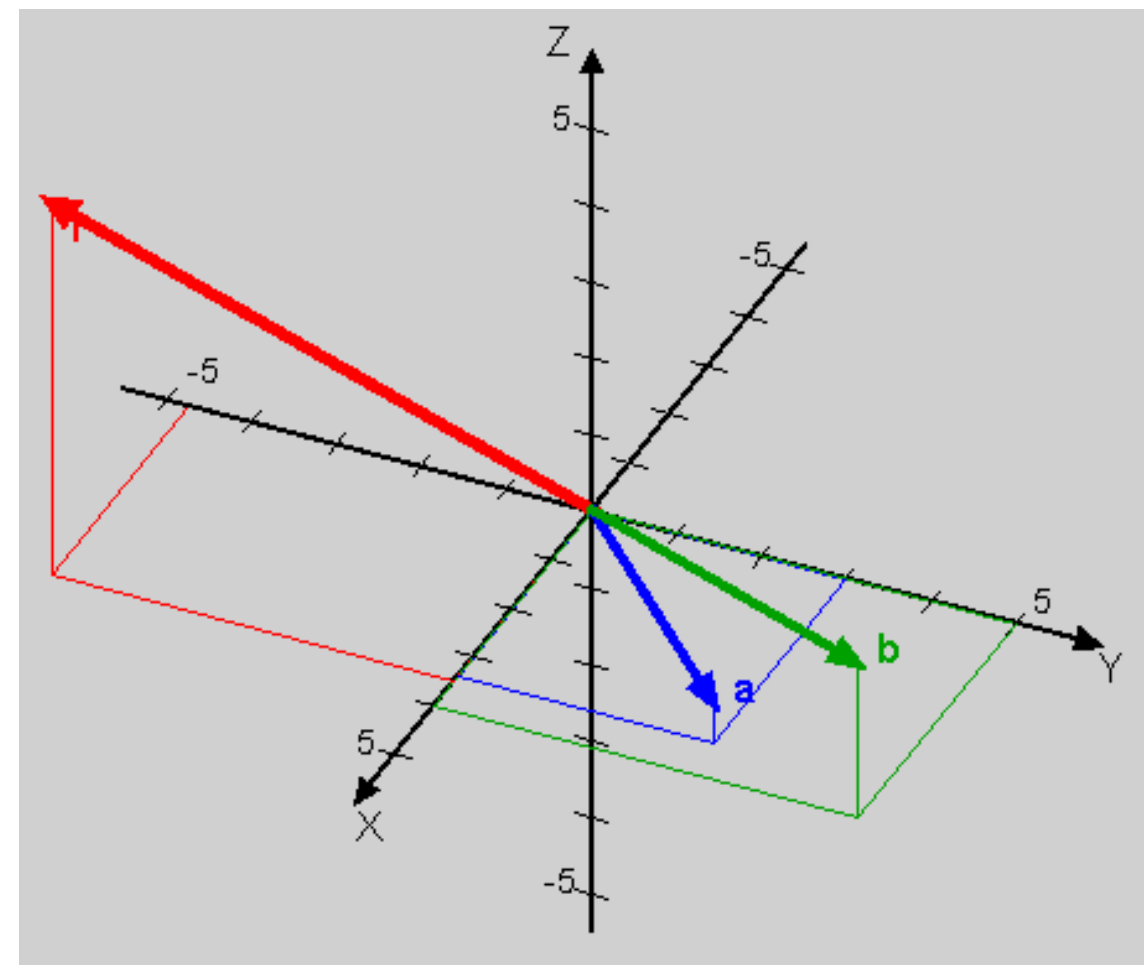

Fig. 4. Visualización del producto vectorial $\mathbf{a} \times \boldsymbol{b}$

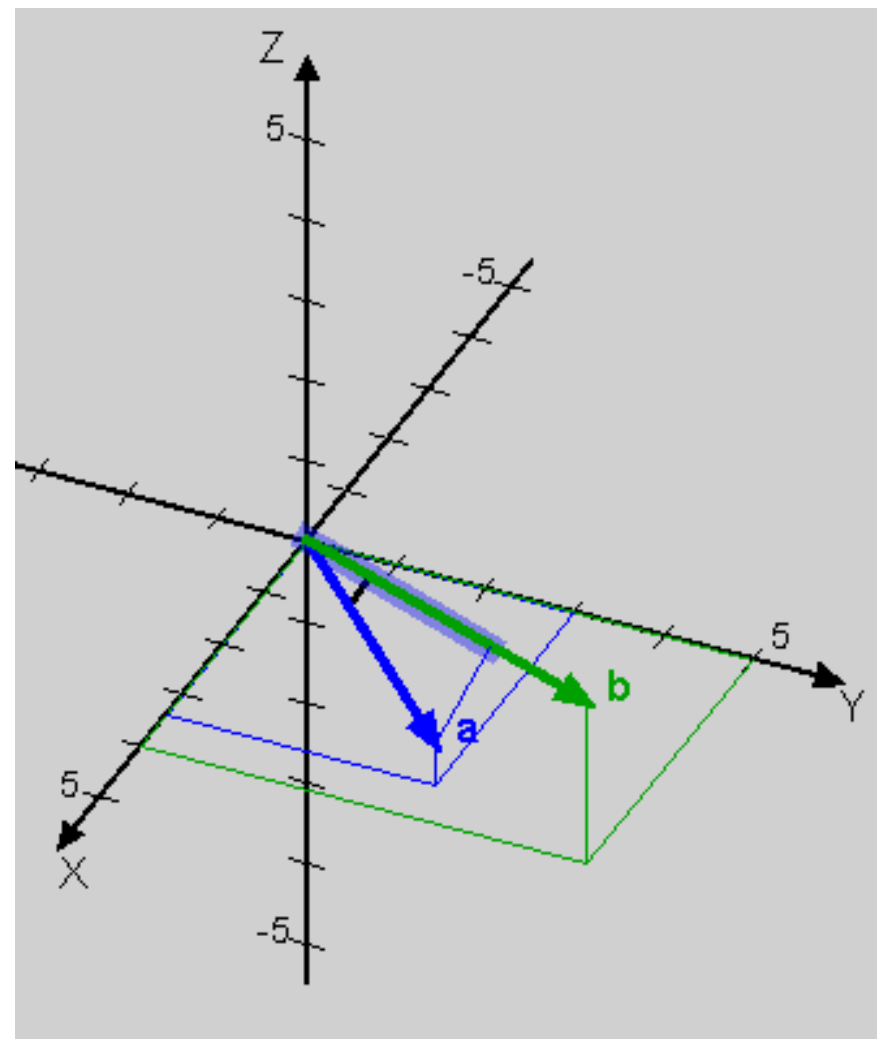

Fig. 5. Visualización de la proyección de $\boldsymbol{a}$ sobre $\boldsymbol{b}$ y del ángulo entre los vectores.

(cc)) EY-NC-ND 2017, Universitat Politècnica de València 
El panel "Valores de interés" (Figura 3) muestra los valores de cuatro propiedades geométricas relevantes: el ángulo entre dos vectores, la proyección de uno sobre otro, el área del paralelogramo que definen, y el volumen del paralelepípedo constituido por tres vectores. Utilizando los correspondientes botones de ojo abierto/cerrado, puede activarse o desactivarse la representación gráfica correspondiente. Como ejemplo, la Figura 5 muestra el ángulo entre vectores, y la proyección de uno sobre otro, mientras que en la Figura 6 se representa el paralelogramo definido por dos vectores y el paralelepípedo constituido por tres de ellos.
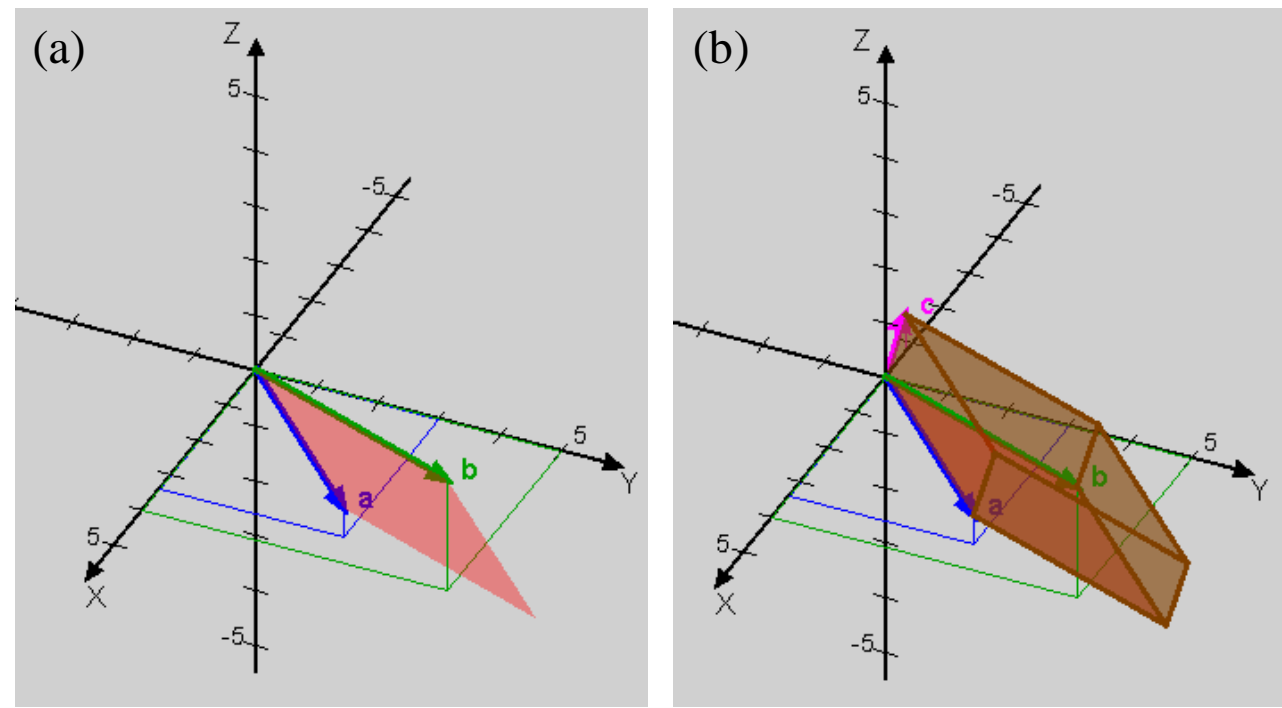

Fig. 6. (a) Paralelogramo definido por dos vectores; (b) paralelepípedo definido por tres vectores.

El panel "Visualización" permite al estudiante controlar diversas opciones de representación. Por ejemplo, la lista desplegable "Suma/resta" establece la forma de representar gráficamente la correspondiente operación (Figura 7), bien mediante la regla del paralelogramo, bien como aplicación sucesiva de los operandos.
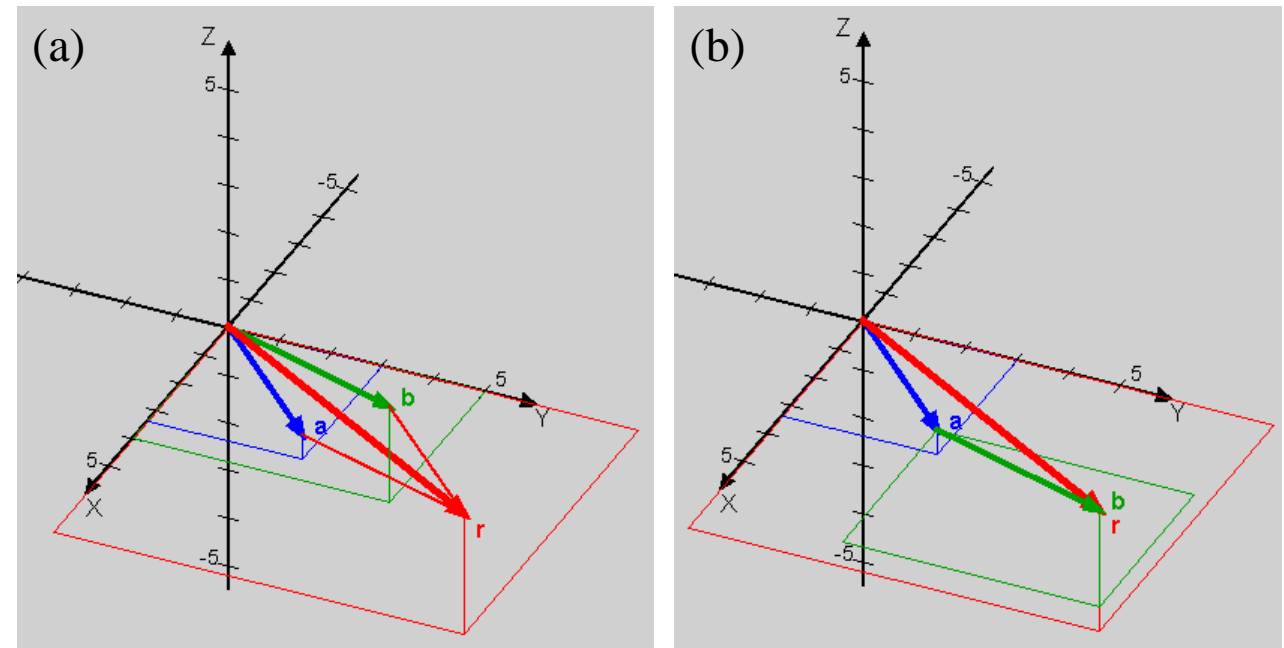

Fig. 7. Suma de vectores: (a) regla del paralelogramo; (b) secuencia de vectores. 


\section{Resultados}

\subsection{Método de evaluación}

Con el objetivo de valorar la influencia del uso del laboratorio virtual de Vectores sobre el aprendizaje de los alumnos, durante el presente curso académico (16/17) se han elegido dos grupos de control. Los dos grupos pertenecen al primer curso de dos títulos de grado de la Escuela Técnica Superior de Ingeniería del Diseño de la Universitat Politècnica de València y a dos asignaturas en las que figura en sus programas el tema de Vectores. En el grupo de la asignatura "Complementos de Física" del grado en Ingeniería Mecánica se impartió el tema de Vectores de forma clásica, y en el grupo de la asignatura "Física Básica" del grado en Ingeniería Eléctrica se utilizó el laboratorio virtual.

Para poder analizar el efecto del uso del laboratorio virtual sobre el rendimiento académico de los estudiantes se realizaron unas pruebas, de respuesta numérica abierta, mediante la utilización de la herramienta de examen de PoliformaT. Con este fin se realizó un primer examen para establecer el nivel de conocimiento de los estudiantes, sobre el tema de vectores, en el punto de partida. Posteriormente, una vez que el tema se había impartido con cada uno de los procedimientos, se volvió a realizar otro examen utilizando la misma plataforma. Asimismo, para conocer cuál era el grado de satisfacción del alumno respecto de la utilización del laboratorio virtual se realizó una encuesta anónima. La encuesta planteaba siete cuestiones y las respuestas fueron valoradas con cinco niveles (TDA: totalmente en desacuerdo, DA: en desacuerdo, IND: indiferente, A: de acuerdo y TA: totalmente de acuerdo).

\subsection{Test de rendimiento académico}

En primer lugar, al comparar los resultados obtenidos en las pruebas realizadas antes de impartir el tema encontramos que el valor medio de las puntuaciones era muy similar en los dos grupos: en "Física Básica" con una media de 5,4 y una desviación estándar de 2,5 y en "Complementos de Física" con una media de 5,2 y una desviación estándar de 2,9. Tal y como era de esperar, después de impartir el tema las puntuaciones medias de los exámenes mejoraron de forma ostensible en los dos grupos. En "Física Básica" la nueva media fue 9,1 con una desviación estándar de 1,5; en "Complementos de Física" la nueva media fue 8,3 y la desviación estándar 1,9. En las gráficas mostradas en la Figura 8, se aprecia cómo las puntuaciones de los exámenes fueron en ambos casos claramente superiores después de impartir el tema, y también menos dispersas. Sin embargo, se observa que en el grupo que utilizó el laboratorio virtual, un porcentaje mayor de alumnos obtuvo la puntuación más alta. De hecho, la puntuación media de los alumnos del grupo que utilizó el laboratorio virtual mejoró un $68 \%$ frente al $60 \%$ del grupo que empleó el método clásico.

No obstante, dado que el desarrollo normal del curso no permitió que las circunstancias en las que se realizaron los exámenes fueran exactamente las mismas para los dos grupos, no podemos afirmar que la mejoría de los resultados observada en el grupo que utilizó el laboratorio virtual sea atribuible completamente a este último. Por esta razón, se realizó una encuesta de opinión, cuyos resultados se analizan en el apartado siguiente.

(cc)) BY-NC-ND 2017, Universitat Politècnica de València 


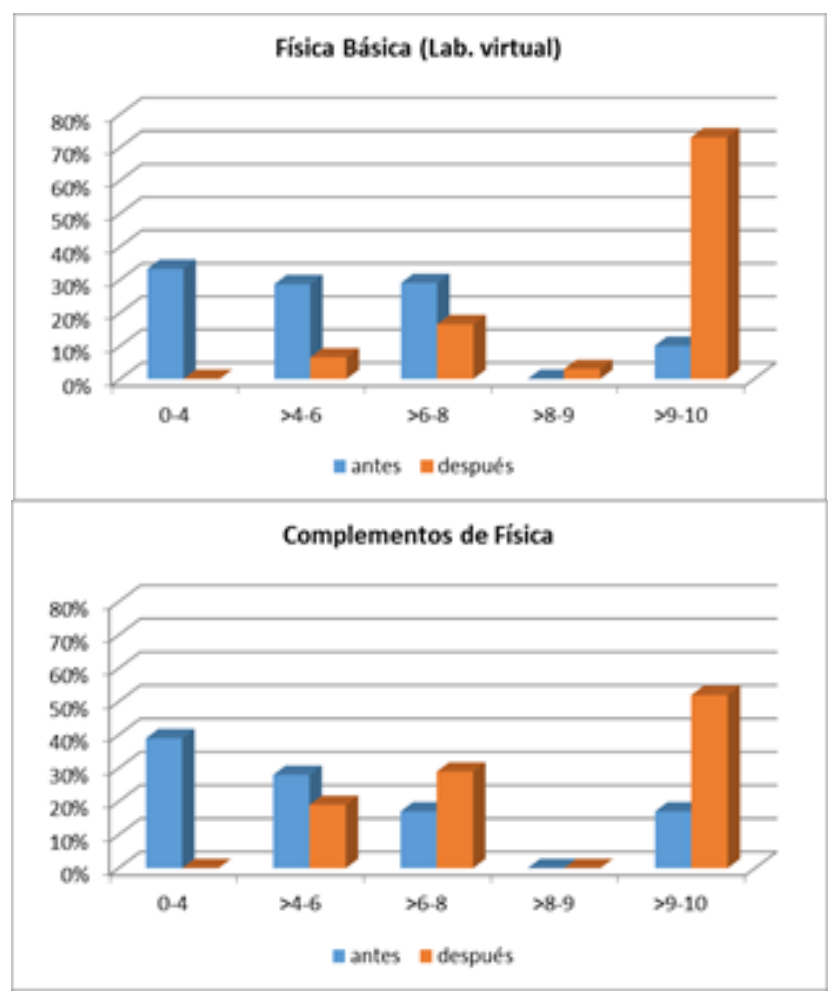

Fig. 8. Representaciones gráficas del porcentaje de alumnos que han obtenido una determinada calificación en los exámenes de cada uno de los grupos, antes y después de impartir el tema.

\subsection{Encuesta de opinión}

La encuesta se realizó, obviamente, en el grupo de estudiantes que había utilizado el laboratorio virtual. En la Tabla 1 se muestran las preguntas y las respuestas obtenidas sobre una muestra de 30 alumnos.

Las respuestas de las cuestiones que están relacionadas con la manejabilidad del laboratorio virtual nos dicen que más del $75 \%$ de los estudiantes exploró las opciones que esta herramienta ofrece (Q6), y el $90 \%$ encontró que el manejo del laboratorio virtual era intuitivo y sencillo (Q2).

La percepción del estudiante sobre la mejoría experimentada en la comprensión del tema de vectores, después de haber utilizado el laboratorio virtual, es también muy positiva ya que el $83 \%$ manifiesta haber mejorado (Q4) y que le ha sido útil (Q1).

Dada la importancia de la motivación en el proceso de aprendizaje se analiza la pregunta Q5, que está vinculada con este factor. Esta cuestión trata de indagar sobre el efecto motivador del laboratorio virtual comparado con el material aportado por el profesor de una forma más tradicional, como apuntes, notas, diapositivas, etc. En la Figura 9 se muestra mediante un gráfico qué porcentaje de los estudiantes respondió en cada uno de los cinco niveles utilizados para valorar la pregunta. Observamos que un porcentaje muy elevado, el 
$83 \%$, encontró más motivadora la utilización del laboratorio frente a un $17 \%$ que o bien no lo consideró así en absoluto, 7\%, o no apreció diferencia, 10\%.

Tabla 1. Preguntas y resultados de la encuesta de opinión.

\begin{tabular}{|l|l|c|c|c|c|c|}
\hline Pregunta & TDA & DA & IND & A & TA \\
\hline Q1 & $\begin{array}{l}\text { Me ha resultado útil el laboratorio virtual de } \\
\text { "Vectores". }\end{array}$ & 2 & 3 & 10 & 15 \\
\hline Q2 & $\begin{array}{l}\text { Me ha resultado fácil e intuitivo el manejo del } \\
\text { laboratorio virtual de "Vectores". }\end{array}$ & 0 & 3 & 7 & 20 \\
\hline Q3 & $\begin{array}{l}\text { En general, mi nivel de compresión de los conceptos } \\
\text { antes de utilizar el laboratorio virtual de "Vectores" era } \\
\text { bueno. }\end{array}$ & 0 & 5 & 4 & 14 & 7 \\
\hline Q4 & $\begin{array}{l}\text { Mi nivel de compresión de los conceptos después de } \\
\text { utilizar el laboratorio virtual de "Vectores" ha mejorado } \\
\text { respecto a mi nivel previo. }\end{array}$ & 0 & 3 & 2 & 21 & 4 \\
\hline Q5 & $\begin{array}{l}\text { Me resulta más motivador repasar el tema "Vectores" } \\
\text { utilizando el laboratorio virtual de "Vectores", que } \\
\text { revisar el material proporcionado por el profesor } \\
\text { (apuntes, notas, diapositivas...) }\end{array}$ & 2 & 0 & 3 & 11 & 14 \\
\hline Q6 & $\begin{array}{l}\text { Cuando he utilizado el laboratorio virtual de "Vectores" } \\
\text { he explorado muchas de las opciones que ofrece. }\end{array}$ & 0 & 0 & 7 & 10 & 13 \\
\hline Q7 & $\begin{array}{l}\text { Mi valoración general del laboratorio virtual de } \\
\text { "Vectores" es buena. }\end{array}$ & 0 & 0 & 3 & 10 & 17 \\
\hline
\end{tabular}

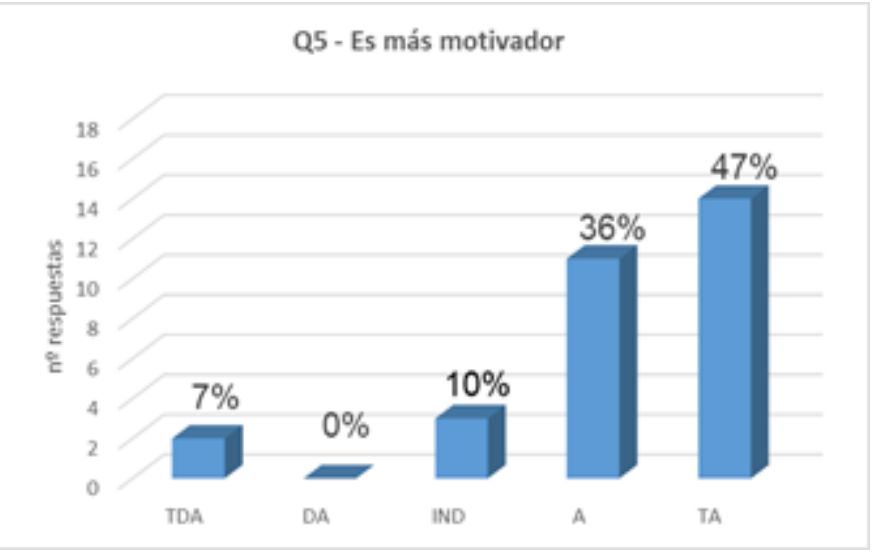

Fig. 9. Representación gráfica del porcentaje de estudiantes que respondió en cada uno de los cinco niveles de la pregunta Q5.

La pregunta Q7 da respuesta a la valoración general del laboratorio. Como se puede observar en el gráfico de la Figura 10, la valoración de los estudiantes es muy positiva ya que el $90 \%$ estuvo de acuerdo o totalmente de acuerdo con la afirmación de que es bueno. 


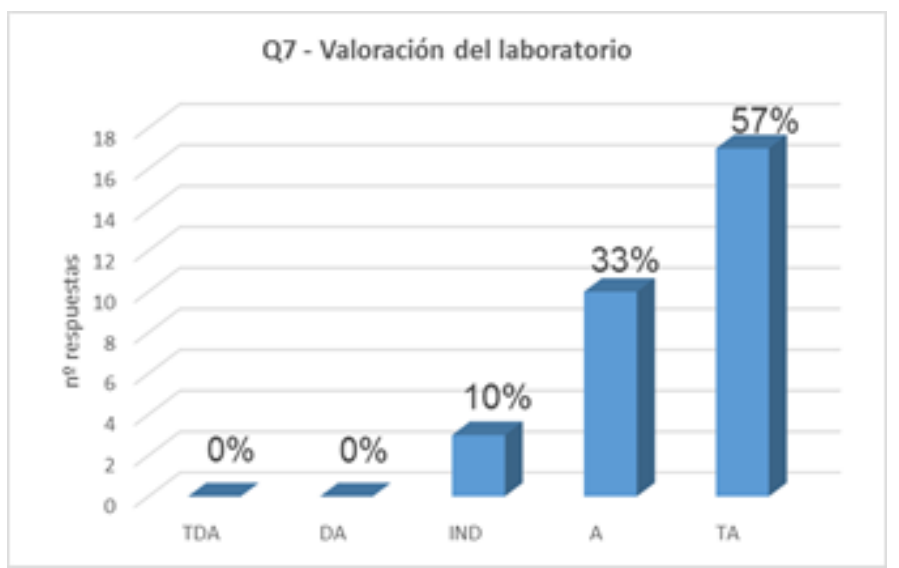

Fig. 10. Representación gráfica del porcentaje de estudiantes que respondió en cada uno de los cinco niveles de la pregunta $Q 7$.

\subsection{Competencias transversales}

Este laboratorio proporciona a los estudiantes los medios para desarrollar su propia comprensión sobre los vectores y sus operaciones. El profesor actúa como guía, dando a los estudiantes las herramientas y mostrándoles las diferentes formas en que pueden aplicarse al proceso de aprendizaje. De este modo, el laboratorio virtual que presentamos sirve de herramienta para la obtención de varias competencias clave transversales (http://www.upv.es/contenidos/COMPTRAN/index-en.html), que ayudarán al alumno a tener una formación más completa para su futuro profesional. El estudiante debe:

- Demostrar la comprensión e integración de los conocimientos, tanto en la propia especialización como en otros contextos más amplios (CT1 - comprensión e integración).

- Aplicar los conocimientos a la práctica, atendiendo a la información disponible y estableciendo el proceso a seguir para alcanzar los objetivos con eficacia y eficiencia (CT2 - aplicación y pensamiento práctico).

- Realizar tareas que requieren procesos de razonamiento más o menos complejos y, en muchos casos, no simplemente una acción asociativa y de rutina. También debe identificar y definir los elementos significativos de un problema para su análisis y resolución efectivos (CT3 - análisis y resolución de problemas).

- Desarrollar un pensamiento crítico, interesado en las bases sobre las que se basan las ideas, acciones y juicios, tanto propios como ajenos. Esto implica cuestionar las suposiciones que subyacen a nuestras formas habituales de pensar y actuar (CT9 - pensamiento crítico).

- Utilizar el aprendizaje de manera estratégica, autónoma y flexible, para manipular el conocimiento, actualizarlo y seleccionar lo que es apropiado para un contexto específico en función del objetivo perseguido (CT11 - aprendizaje permanente). 
El estudiante debe prepararse para enfrentar situaciones complejas acomodando los recursos disponibles. El uso de estas herramientas (laboratorios virtuales) promueve la autonomía y la iniciativa. El estudiante aprende y aplica conocimientos y habilidades de manera autónoma.

\section{Conclusiones}

Este trabajo presenta un laboratorio virtual diseñado para ayudar a los estudiantes a profundizar en las operaciones con vectores y sus propiedades. El uso de esta herramienta, que permite introducir diferentes vectores, visualizar su representación 3D y operar con ellos, ofrece una aproximación sencilla y completa al cálculo vectorial. La encuesta de satisfacción mostró un alto grado de motivación en los alumnos que usaron esta herramienta, que se vio reflejado en un aumento significativo del porcentaje de alumnos con nota más alta.

Las competencias transversales vinculadas al uso de este laboratorio virtual han sido enumeradas destacando los aspectos docentes más relevantes de este recurso.

\section{Agradecimientos}

Los autores desean agradecer al Instituto de Ciencias de la Educación de la Universitat Politècnica de València (UPV) su apoyo en los grupos de innovación docente MoMA y e-MACAFI, y por subvencionar el proyecto PIME B24. J.A. Sans agradece el apoyo financiero del programa de becas "Ramón y Cajal", y al Instituto de Diseño para la Fabricación y Producción Automatizada de la UPV por permitirle conseguir una venia docendi en el Departamento de Física Aplicada de la UPV.

\section{Referencias}

CHRISTIAN, W. y BELLENI, M. (2001). Physlets: Teaching physics with interactive curricular material. Upper Saddle River, NJ, USA: Prentice Hall.

ESQUEMBRE, F. (2002). "Computers in physics education" en Computer Physics Communications, vol. 147 , p. 13-18.

ESQUEMBRE, F. (2004). "Easy Java Simulations : a software tood to create scientific simulations in Java" en Computer Physics Communications, vol. 156, p. 199-204.

JIMOYIANNIS, A. y KOMIS, V. (2001). "Computer simulations in physics teaching and learning" en Computer \& Education, vol. 36, p. 183-204.

STEINBERG, R.N. (2000). "Computers in teaching science: To simulate or not to simulate" en American Journal of Physics, vol. 68, p. S37-S41.

VIDAURRE, A., RIERA, J., GIMÉNEZ, M.H. y MONSORIU, J.A. (2002). "Contribution of digital simulation in visualizing physics processes" en Computer Applications in Engineering Education, vol. 10, p. 45-49.

(c)) EY-NC-ND 2017, Universitat Politècnica de València 